\title{
Endoscopic Endonasal Surgery for Two Cases of Giant Nasal Lobular Capillary Hemangiomas
}

\author{
Jeong Hwan Choi ${ }^{\mathrm{a}, \mathrm{b}}$, Kyoungrai Cho
}

\begin{abstract}
Lobular capillary hemangioma $(\mathrm{LCH})$ is a benign lesion originating in the vascular tissue of skin and mucosa. LCH rarely involves nasal cavity (LCHNC). Complete surgical excision is the treatment of choice. The endoscopic endonasal surgery (EES) technique can achieve good cosmetic results by avoiding external incision. In a patient with a rapidly growing intranasal lesion, $\mathrm{LCH}$ should be considered as a differential diagnosis. Rapid growth, epistaxis, and its gross findings often mimic malignancy. Histologic confirmation is inevitable. In this report, we present two patients with huge $\mathrm{LCH}$ and discussed the clinical, radiological and histological characteristics of LCHNC, and its treatment.
\end{abstract}

Keywords: Lobular capillary hemangioma; Pyogenic granuloma; Endoscopic endonasal surgery; Nasal cavity; Epistaxis

\section{Introduction}

Lobular capillary hemangioma ( $\mathrm{LCH}$, also known as a pyogenic granuloma, $\mathrm{PG}$ ), is a benign capillary proliferation of unknown etiology. LCH usually involves the gingiva, lips, tongue, and buccal mucosa. However, it is obscure to many rhinologists, because the nasal cavity is a relatively rare location for this lesion. The most common symptoms are epistaxis and nasal obstruction. The treatment of choice is endoscopic endonasal surgery (EES) even for large lesion. We discussed the differences between the cases in terms of management processes and results.

\footnotetext{
Manuscript accepted for publication September 24, 2012

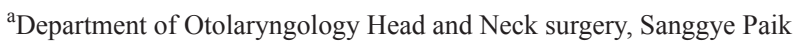
Hospital, College of Medicine, Inje University, Seoul, Korea

${ }^{\mathrm{b}}$ Corresponding author : Jeong Hwan Choi, Department of Otolaryngology Head and Neck surgery, Sanggye Paik Hospital, College of Medicine, Inje University, 761-1 Sanggye-7-dong

Nowon-gu, Seoul, 139-707, Republic of Korea (South).

Email: choijh92@paik.ac.kr
}

doi:http://dx.doi.org/10.4021/jmc873w

\section{Case Report}

\section{Case 1}

A 29-year-old female was referred by her obstetrician immediately after childbirth. She has a large exophytic mass in her right nasal cavity. She also reported a two-month history of progressive nasal obstruction and intermittent epistaxis which had been requied multiple nasal packing. Nasal endoscopic examination revealed a large smooth-surfaced hemorrhagic polypoidal mass totally occluding the right nasal cavity. The mass was soft, mobile, nonpulsatile, and nontender. The surface of the lesion was friable, and manipulation elicited active bleeding. The postnasal space was clear and rest of the head and neck examination did not reveal any abnormalities. Incisional biopsy was not performed due to probablility of significant hemorrhage. Computed tomography of the paranasal sinuses (PNS CT) demonstrated a $3 \times 5$ $\mathrm{cm}$ sized soft tissue opacity lesion with intense enhancement occupying the entire right nasal cavity (Fig. 1A, B). The lesion was well circumscribed without any evidence of bony destruction or periostial new bone formation. She wanted prompt excision without observation or medication because of long standing disturbing symtoms. Thereupon, under general anesthesia, she underwent endoscopic exploration of the right nasal cavity with preparations for possible lateral rhinotomy for the excision of this tumor. The operation was begun with debulking of tumoral tissue that had not involved the nasal mucosa by soft tissue shaver. This allows better visualization, provides enough space for determination of the original site. The mass was found to be originating from the posterior portion of the right inferior turbinate. Despite significant bleeding, under the endoscopic guidance complete excision of the mass was perfectly done due to the excellent exposure of the lesion, provided by the magnified telescopic view and suction and bipolar cautery to ensure hemostasis. The surgery involved partial resection of the inferior turbinate to remove a cuff of normal appearing tissue. The operation was finished without requirement of perioperative blood transfusion. The patient had an uneventful postoperative course, and there was no recurrence in the following year. 


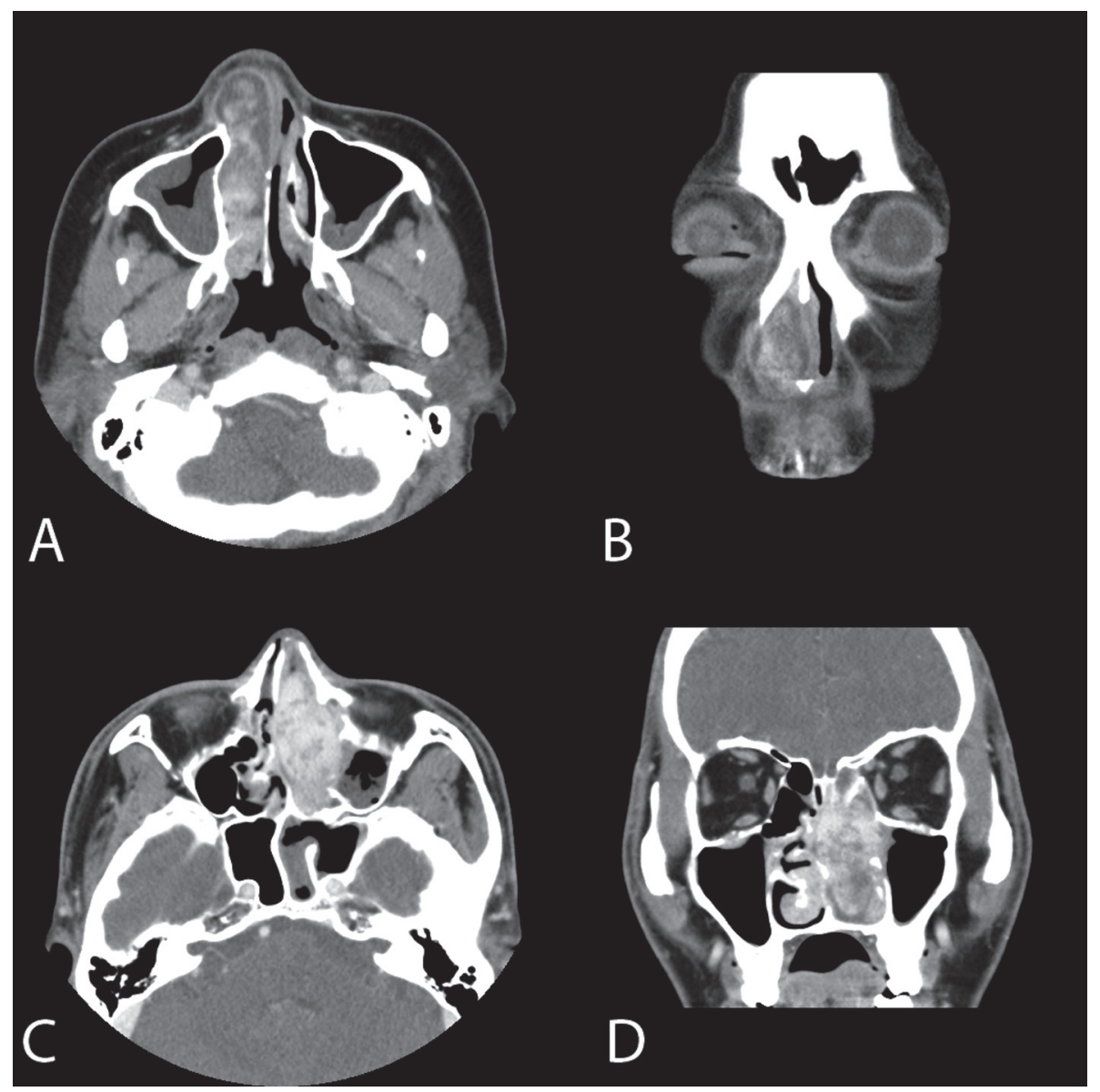

Figure 1. Axial (A) and coronal (B) enhanced computed tomography of the paranasal sinuses (PNS CT) of case 1 demonstrate a $3 \times 5 \mathrm{~cm}$ sized lobuated soft tissue opacity occupying the entire right nasal cavity with intense enhancement. The lesion was well circumscribed without any evidence of bony destruction. Axial (C) and coronal (D) PNS CT of case 2 show a soft tissue opacity with intense enhancement occupying the entire left nasal cavity. The nasal septum was deviated to the right side. However, there is no evidence of bony destruction.

In histopathological examination, multiple polypoid fragments of angiomatous tissue protruding above the surrounding mucosa were seen. The mass was covered with pseudostratified ciliated epithelium showing focal squamous metaplasia and ulceration below which can be seen profound inflammation in the connective tissue. The submucosal lamina propria contained lobular proliferated capillaries with lumina of varying sizes surrounded by an edematous fibromixoid stroma (Fig. 2A ,B). There was no evidence of mitotic activity or malignancy. These histopathological findings were consistent with a LCH.

\section{Case 2}

A 47-year-old male presented with complaints of having had epistaxis and nasal obstruction for the past 3 months. Physical examination revealed reddish hemorrhagic polypoid mass near totally obstructing the left nasal cavity. PNS CT revealed a soft tissue opacity lesion with intense en- hancement occupying the entire left nasal cavity without any evidence of bony (Fig. 1C, D). Any punch biopsy specimen could not be obtained due to significant bleeding even by needle stick for local anesthesia at the mass. Excision of the mass was done under general anaesthesia using an EES technique, following superselective embolization the day before surgery (Fig. 3). At operation field, the shrinked nasal mass by embolization was found. The mass was extirpated by two pieces of $1.7 \times 0.7 \mathrm{~cm}$ sized masses (Fig. 4). The histopathologic findings were consistent with a LCH (Fig. 2C, D).

\section{Discussion}

$\mathrm{LCH}$, a benign, rapidly growing tumorous condition or benign capillary proliferation affects skin and mucous membranes. Occurrence of LCH in human was first originally described in 1897 by Poncet and Dor, who named this lesion botryomycosis hominis [1]. LCH has been referred to by a 


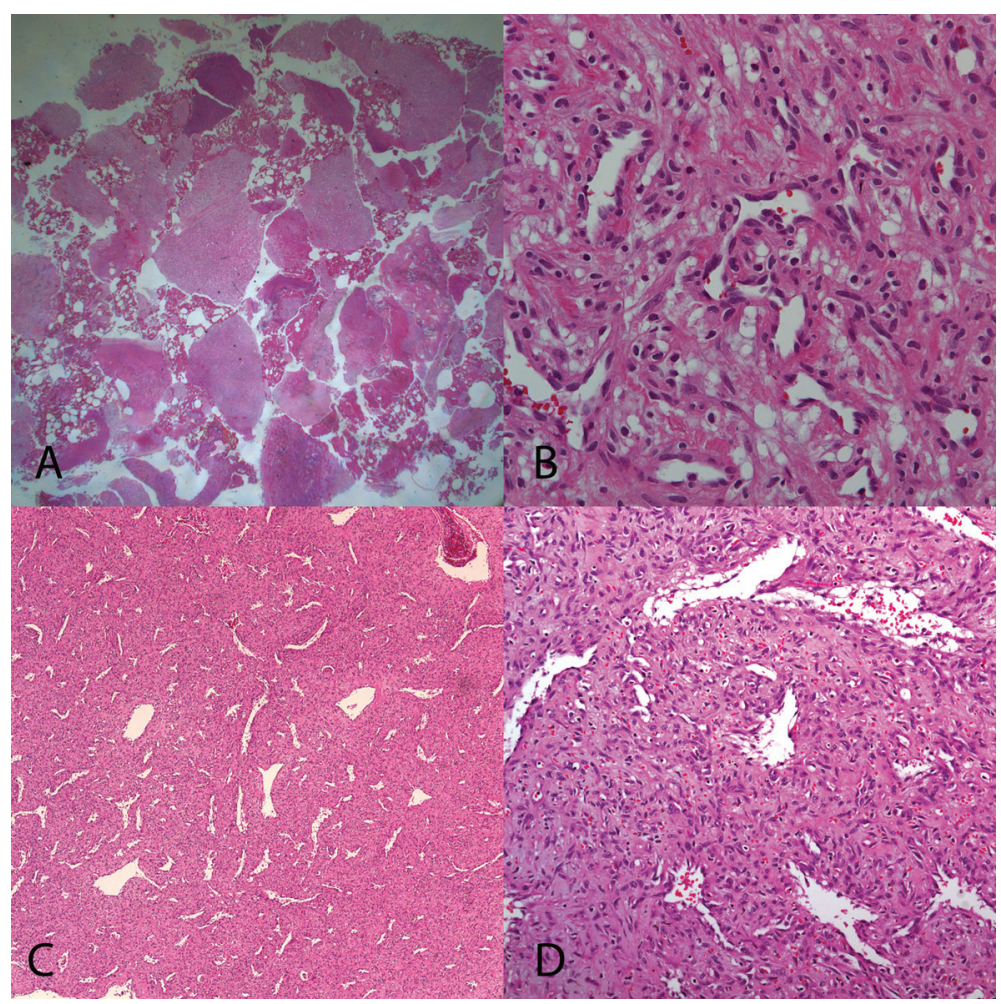

Figure 2. In histopathological examination of case $1(A, B)$ and case 2 (C, D), multiple polypoid fragments of angiomatous tissue protruding above the surrounding mucosa were seen. The mass was covered with pseudostratified ciliated epithelium showing focal squamous metaplasia and ulceration below which can be seen profound inflammation in the connective tissue. The submucosal lamina propria contained lobular proliferated capillaries with lumina of varying sizes surrounded by an edematous fibromixoid stroma. There was no evidence of mitotic activity or malignancy. These histopathological findings were consistent with a lobular capillary hemangioma LCH. (A, C: H \& E, × 40, B, D: H \& E, × 200).

variety of other names such as PG, granuloma gravidarum, pregnancy tumor, hemangiomatous granuloma, granuloma telangiectacticum, granuloma pediculatum benignum, benign vascular tumor, and Crocker and Hartzell's disease [2]. However, some researchers believe that Hartzell in 1904 introduced the term "PG" that is widely used in the literature, although, it is a misnomer because clear histologic and microbiologic features indicating an infectious origin are lacking. In addition, the term "granuloma" would allude to the presence of granulation tissue in the lesion, which is not

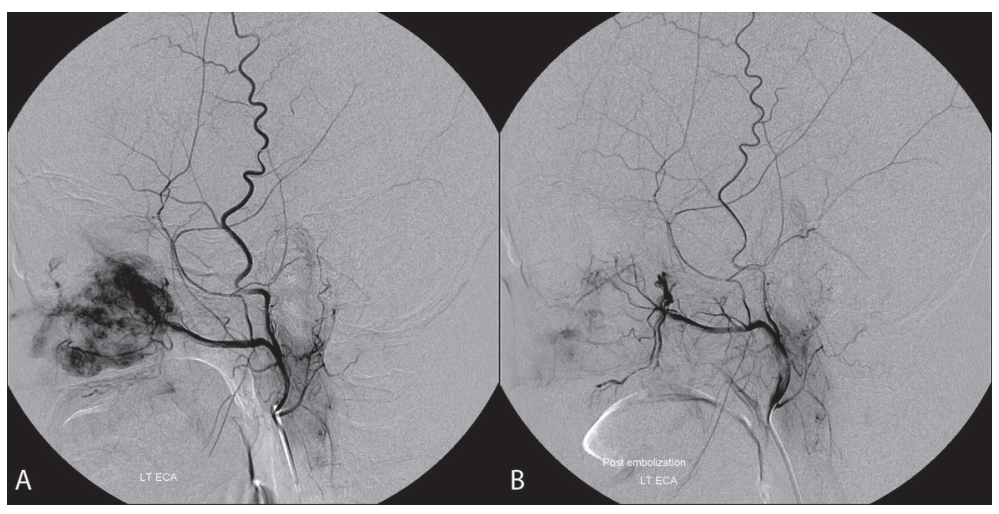

Figure 3. Carotid angiogram of nasal mass in case 2 shows mass (arrow) lesion staining in the left nasal cavity, supplied by internal maxillary artery of left external carotid artery (A). After successful embolization by polyvinyl alcohol, no more staining lesion was observed (B). 


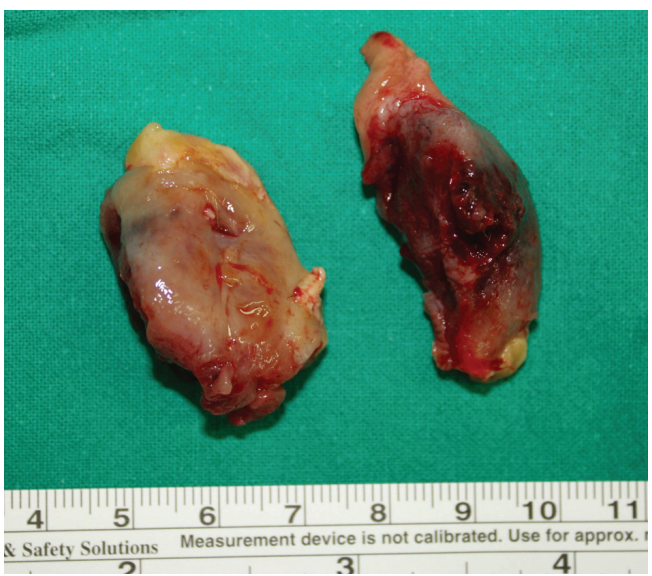

Figure 4. The shrinked nasal mass by embolization was extirpated by two pieces of $1.7 \times 0.7 \mathrm{~cm}$ sized masses.

typical of a LCH. Mills suggested that the accurate, descriptive term for PG is LCH because it emphasizes the essential component of the lesion, namely, a circumscribed aggregate of capillaries arranged in one or more lobules [3]. Nevertheless, PG is still the more popular terminology [1].

The peak prevalence is in teenagers and young adults, with a female predilection of 2:1 [4]. Most authors list the gingiva, lips, tongue, buccal mucosa and palate as the most commonly involved locations. LCH rarely involves nasal cavity (LCHNC) which most commonly manifests as progressive nasal obstruction and epistaxis. The first case of LCHNC was described in 1940 [5]. The precise mechanism for the development of LCH is unknown. Traumas (habitual nose picking, nasal packing) and hormonal influences (pregnancy, oral contraceptives) have been suspected to act in the pathogenesis [6].

LCHNC is rarely discovered as an incidental finding, since epistaxis and nasal obstruction will soon bring the patient to a physician. LCHNC may rarely present as a mass of considerable size and thus entirely fill the nasal cavity; such lesions have been termed as giant LCH $[6,7]$. Nasal examination reveals a fragile and usually pedunculate, pinkred mass which has irregular borders [3]. Significant bleeding occurs after biopsy because of the vascular structure of the mass [7].

On PNS CT, LCHNC appears as a non-specific unilateral mass of soft tissue density [6]. Some rare cases of bony erosion due to compression have been described [8]. Angiography typically shows increased vascularity in the area of the tumor.

Histologically, the LCHNC lesion consists of 2 areas: a lobular and a superficial ulcerative area. The lobular area is characterized by capillary proliferation with a microscopically distinct lobular architecture. The superficial portion of the lesion may undergo secondary nonspecific changes, including stromal edema, capillary dilation, inflammation, and a granulation tissue reaction.

The diagnosis may be delayed because of the overlapping of the symptoms with chronic sinusitis. Furthermore, these tumors can be difficult to distinguish from a malignant lesion due to their rapidity of growth and presentation with epistaxis. A differential diagnosis of a hypervascular mass of the nasal cavity in patients with nasal obstruction and epistaxis might include a diverse range of congenital, inflammatory and neoplastic processes, among which are; polyps, rhinosporidiosis, sarcoid, Wegener's granulomatosis, meningoencephalocoele, glioma, cavernous hemangioma, cherry angioma, hemangiopericytoma, histiocytoma, leiomyoma, osteoma, squamous cell carcinoma, adenocarcinoma, juvenile nasopharyngeal angiofibroma, Kaposi's sarcoma, esthesioneuroblastoma, angiosarcoma, extranodal NK/T-cell lymphoma, and hypervascular metastases, particularly from kidney, thyroid, lung, or breast. In children, there is always the possibility of a foreign body.

Common treatments for LCH are curettage, cryotherapy, and excision. Excision of the entire lesion has been the treatment of choice. Promising results have been reported for various new treatments, such as laser therapy and sclerosing agents [9]. The EES provides better visualization of the mass and surrounding anatomy, thus allowing the surgeon to remove the mass completely without an external incision. The recurrence rate after excision is approximately $15 \%$ [2]. Recurrence is usually due to incomplete excision [9]. No malignant transformations have been reported [10].

To summarize the lesson from two cases; 1) LCH would be more appropriate term to remind clinician characteristics of the hemangioma. PG might wrongfully remind unexperienced doctor granulation not hemangioma which needed preoperative embolization; 2) Preoperative embolization is highly recommended. Embolization cannot only decrease the amount of bleeding but also decrease the size of mass; 3 ) The EES is recommended for even giant LCHNC. The EES can provide excellent exposure of the lesion and achieve the good cosmetic results.

In conclusion, giant $\mathrm{LCH}$ is a rare lesion of unknown etiology. It should be considered in the differential diagnosis of rapidly enlarging vascular lesions within the nasal cavity. THE EES is the appropriate treatment even for extremely large lesions.

\section{Conflict of Interest}

None.

\section{References}

1. Sheen TS, Ko JY, Hsu YH. Pyogenic granuloma--an uncommon complication of nasal packing. Am J Rhinol. 
1997;11(3):225-227.

2. Bhaskar SN, Jacoway JR. Pyogenic granuloma--clinical features, incidence, histology, and result of treatment: report of 242 cases. J Oral Surg. 1966;24(5):391-398.

3. Mills SE, Cooper PH, Fechner RE. Lobular capillary hemangioma: the underlying lesion of pyogenic granuloma. A study of 73 cases from the oral and nasal mucous membranes. Am J Surg Pathol. 1980;4(5):470479.

4. Ramirez K, Bruce G, Carpenter W. Pyogenic granuloma: case report in a 9-year-old girl. Gen Dent. 2002;50(3):280-281.

5. Frank I, Blahd M. Pyogenic granuloma of the nasal fossa. Arch Otolaryngol Head Neck Surg 1940;31(6):919924.

6. Puxeddu R, Berlucchi M, Ledda GP, Parodo G, Farina
D, Nicolai P. Lobular capillary hemangioma of the nasal cavity: A retrospective study on 40 patients. Am J Rhinol. 2006;20(4):480-484.

7. Bhattacharyya N, Wenokur RK, Goodman ML. Endoscopic excision of a giant pyogenic granuloma of the nasal cavity caused by nasal packing. Rhinology. 1997;35(1):44-45.

8. Lance E, Schatz C, Nach R, Thomas P. Pyogenic granuloma gravidarum of the nasal fossa: CT features. J Comput Assist Tomogr. 1992;16(4):663-664.

9. Zarrinneshan AA, Zapanta PE, Wall SJ. Nasal pyogenic granuloma. Otolaryngol Head Neck Surg. 2007;136(1):130-131.

10. Miller FR, D’Agostino MA, Schlack K. Lobular capillary hemangioma of the nasal cavity. Otolaryngol Head Neck Surg. 1999;120(5):783-784. 